\title{
Efficacy of Bisphosphonates and Recombinant Parathyroid Hormone in Treatment of Osteoporosis in Pakistan
}

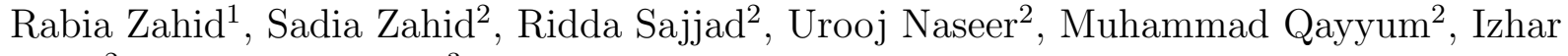 \\ $\mathrm{Ullah}^{2}$, and Ejaz Rafique ${ }^{3}$ \\ ${ }^{1}$ Government College University Faisalabad \\ ${ }^{2}$ University of the Poonch Rawalakot \\ ${ }^{3}$ The University of Lahore
}

October 20, 2020

\begin{abstract}
Background; Osteoporosis is one of such disease which causes bone mass reduction by decreased formation of new bones. Low bone mineral density is frequent factor for osteoporosis. Increased osteoclast life span and advanced osteocytes as well as osteoblasts apoptosis is well adopted pathophysiological mechanism for low bone mass. Women are more prone than man. Approximately 200 million women have osteoporosis worldwide. Objective; Therefore, this study is designed to compare efficacy of bisphosphonates and recombinant human Para-thyroid hormone in treatment of osteoporosis. Main outcome measures; The comparison of these two treatment strategies is based upon therapeutic effectiveness, cost of therapy and availability of the drugs. Methods; The total duration of study was 1 year and during the study period a total 60 samples were analyzed randomly. Data was collected using a well-designed questionnaire. Results; rhPTH increase bone mass density (BMD) by 8-9\% while bisphosphonates and estrogen therapy increase BMD 3-5\% during the same time period. Conclusion; The current study was conducted in local population so that we could offer them the test possible treatment among the two most common treatment therapies available for osteoporosis.
\end{abstract}

\section{INTRODUCTION}

Osteoporosis, a skeletal disease having characteristic bone mass reduction and bone micro architecture deterioration including bone fragility and high risk of fractures. Fractures are more prone due to decrease in bone mineral density and has some importance in both men and women ${ }^{[1]}$. Osteoporosis has become major health problem with the advancement in socioeconomical conditions and delay of humanoid life span. Bone mass maintained by bone resorption and dynamic balance of bone formation, when lost results in osteoporosis $^{[2]}$.

Fastest growing population in the world are elderly age, bone mass decline and risk of fractures increases as age increases. As population longevity in developing countries continues to increase likelihood of osteoporosis will increase in these countries [3]. The prevalence of osteoporosis that is reported in women in United Kingdom, France and Germany, United States of America, and Japan, as 9\%, 15\%, 16\% and 38\% respectively; whereas in men, United Kingdom, Japan, Canada and France as 1\%, 4\%, 3\%, 8\% respectively. In 2000, around 9.0 million osteoporotic fractures were stated, 1.6, 1.7 and 1.4 million of which found at the hips, forearm and vertebral fractures ${ }^{[4]}$. It was concluded that in Pakistan there are 9.90 million osteoporotic patients (7.19 million are women and 2.7 million are male) and these numbers will increase to 11.3 million in 2020 and 12.9 million in 2050; after conducting the large countrywide study using the quantitative ultrasound in Pakistan ${ }^{[5]}$. 
In osteoporosis patients, care of patients and underlying drug development continues to improve with increased understanding of bone loss. In a co-ordinated and continuous fashion old bones are removed by osteoclast and new bones are formed by osteoblast and process is called bone remodeling. With certain diseases and aging among old and new bone incomplete replacement occurs ${ }^{[6]}$. A new mechanism for osteoporosis recently suggested is imbalance between osteoblastogensis and adipogenesis of mesenchymal stem cells of bone marrow. The comprehensiveness and exhaustiveness of this mechanism is still uncertain ${ }^{[7]}$.

Osteoporosis can be classified in two main categories; primary osteoporosis and secondary osteoporosis. Primary osteoporosis can occur both in males and females but occur oftenly in postmenopausal women, also happens in men's life later. Use of medications (glucocorticoid, immunosuppressant's), disease (Cushing's syndrome, hyperthyroidism), other conditions (vitamin D deficiency and hypogonadism) results in secondary ${ }_{\text {osteoporosis }}{ }^{[8]}$. Primary osteoporosis is the most commonly found type of disease and it has sub types as: post-menopausal osteoporosis or type-I and senile osteoporosis or type-II ${ }^{[9]}$. At menopause ovarian function reduces that give consequences as limited estrogen production and parallel increasing follicle stimulating hormone. A significant bone resorption and quick bone loss is result of combined effect of estrogen decrease and increase in FSH; this is the basic for onset of post-menopausal osteoporosis ${ }^{[10]}$.Senile osteoporosis is also called Involutional osteoporosis (type-II) and is related to bone mass loss due to cortical and trabicular bones aging ${ }^{[11]}$. Secondary osteoporosis is characterized by micro architectural bone changes causes fragility fractures accompanied by underlying disease or medication associated with low bone mass ${ }^{[12]}$.Medical conditions or treatments that interfere with the attachment of peak bone mass and/or may predispose to accelerated bone loss results in secondary osteoporosis. Secondary osteoporosis is becoming more frequently diagnosed despite of being less common. It shows similar prevalence among men and women ${ }^{[13]}$.

In osteoporosis major component of fragile bone is low bone mass resulting from: (1) A small peak bone mass at young adult hood. (2) At menopause or later ages as increased bone loss. (3) Combination of both.

Peak bone mass and subsequent bone loss is influenced by many factors that are physical in activity, genetic factors, nutritional factors and hormonal factors ${ }^{[14]}$.Development or appearance of osteoporosis is due to many causes. When cause is natural it is known as primary osteoporosis. Idiopathic osteoporosis develops when cause is unknown ${ }^{[15]}$. Low estrogen level, genetic predispositions, low body weight, smoking, excessive consumption of coffee and long periods of immobilization are contributing factors towards osteoporosis. Deficiency of calcium, 1.25 dihydroxy vitamin D and proteins are dietary factors which increase the risk of osteoporosis ${ }^{[16]}$. Many of the nutrients and food components we consume influence bones by various mechanisms that include bone structure alterations, bone metabolism rate, the endocrine system, homeostasis of calcium and other bone active mineral elements. These dietary factors include inorganic minerals (e.g. calcium, magnesium, phosphorus, sodium, potassium and various trace elements), vitamins (vitamin A, $\mathrm{D}, \mathrm{E}, \mathrm{K}, \mathrm{C}$ and certain $\mathrm{B}$ vitamins) and macronutrients such as proteins and fatty acids increase risk of osteoporosis ${ }^{[17]}$. Osteoporosis is diagnosed clinically or radio graphically. Low impact fractures or fragility fractures may present with osteoporosis. T-score of -1.25 or below as determined by DEXA scan of the total hip, femoral, neck or lumbar spine is most common method for diagnosis of osteoporosis. BMD can be assessed by quantitative computed tomography by radiation exposure and cost has been limited ${ }^{[18]}$.

The whole population must focus on nutritional and life style changes for prevention of osteoporosis. Increase awareness through patient education about the modifiable risks of osteoporosis is an important primary care role ${ }^{[19]}$.As described women (especially post-menopausal) are osteoporotic in high ratio, so preventive measures are taken in women to avoid osteoporosis. Ensuring that women reach their peak bone mass $(\mathrm{PBM})$ and minimize bone loss through their early adult years is primary prevention goal in osteoporosis. In women who have osteoporosis or osteopenia by bone density measurement, fracture prevention is basic focus of secondary prevention. In women who have already sustained fractures in osteoporosis, preventing future fractures is tertiary prevention strategy ${ }^{[20]}$.

By assessment of bone mineral density by DEXA scan in vast majority of cases; those at high risks of osteoporotic fractures should be targeted for treatment. The treatment can be started without measuring BMD in people with two or more vertebral fractures and having high risks of future fractures. Goals of treat- 
ment are fracture prevention, stabilization and achievement of an increase bone mass, relieving fractures and skeletal deformity and physical function minimization ${ }^{[21]}$. Bracing, exercise programs, calcium and vitamin D supplementation, fall prevention and kyphoplasty are non-pharmacological therapies for managing osteoporosis and have significant role in fractures minimization ${ }^{[22]}$.Various pharmacological treatments are also available for treatment of osteoporosis. In alphabetic order, bisphosphonates (alendronate, alendronate plus $\mathrm{D}$, risedronate and zoledronic acid) calcitonin, estrogen (estrogen and/or hormone therapy), estrogen agonists, tissue selective estrogen complex, parathyroid hormone (PTH, teriperatide) and receptor activator of nuclear factor kappa-B, ligand inhibitor denosumab; are current FDA approved pharmacological treatment of osteoporosis $[23]$

Osteoporosis pharmacological treatment comprises of two main classes; antiresorptive agents and anabolic agents. Bone resorption is limited through inhibition of osteoclasts activity by use of anti resorptive agents such as bisphosphonates. Active building of bone mass is promoted using anabolic agents such as parathyroid hormone $^{[24]}$.

Various physiochemical effects on bone salt crystals and biological effects on mineralization and resorption of bones are biological actions of bisphosphonates ${ }^{[25]}$. Bisphosphonates are reported as most potent and effective inhibitors of bone resorption. Extended use of bisphosphonates in all conditions such as excessive osteoclast-mediated bone resorption such as steroid induced osteoporosis has been reported in clinical use ${ }^{[26]}$. Bisphosphonates discovery and development for the treatment of bone diseases is fascinating story extended over three decades. The bone resorption inhibitors bisphosphonates are "P-C-P" structures i.e. two phosphonates groups attached to single carbon atom. Bisphosphonates are therefore stable analogs of naturally occurring pyrophosphonate compounds helpful in intracellular and extra cellular mode of action ${ }^{[27]}$.

Bisphosphonates as anti resorptive agents are most broadly consumed drugs group for osteoporosis. By inhibiting recruitment of osteoclasts bisphosphonates straightaway diminish the count of osteoclasts and also inhibit osteoclast stimulating activity of osteoblasts. In patients with osteoporosis, bisphosphonates normalize bone turn over, reduce number of bone remodeling unit, restore the balance of bone remodeling, prevent bone loss and deterioration of bone structure and reduce fracture risks. ${ }^{[1]}$ In many clinical trials of postmenopausal women suffering osteoporosis, efficacy of bisphosphonates is thoroughly researched and reported. Large number of meta-analysis of bisphosphonates have been performed but recently adverse effects related reviews are found and few reviews on clinical impact in population for future prevention ${ }^{[28]}$. Adverse effect such as gastro esophageal irritation was recognized early on with bisphosphonates therapy. Short term adverse effects include: upper GI adverse effects, acute phase reaction, severe musculoskeletal pain, hypocalcaemia, esophageal cancer with oral use and ocular inflammation. Long term adverse effects include: osteonecrosis of jaw, atrial fibrillation, severe suppression of bone turn over and subtrochanteric femoral fractures ${ }^{[29]}$.

In mammals maintenance of calcium homeostasis is central role played by human parathyroid hormone that is an 84-amino acid peptide. The primary mechanism through which this class of drug operates is inhibition of bone remodeling. Decline in remodeling rate have several beneficial effects such as bone density improvement through decrease in remodeling space size, cancellous bone architecture prevention, decrease in resorption cavities number and cortical porosity decrease ${ }^{[30]}$. Teriperatide is amino-terminal fragment of parathyroid hormone and is a potent bone formation agent. Teriperatide increases the number of osteoblasts and rate of new bone formation by increasing osteoblasts birth rate and preventing osteoblasts apoptosis. Teriperatide is administered parenterally; subcutaneously ${ }^{[31]}$.

Injection site pain and swelling ( $<3.3 \%$ of patients), nausea $(8.5 \%)$, headache $(7.5 \%)$, leg cramps $(2.6 \%)$ and dizziness $(8 \%)$ are most common adverse effects of parathyroid hormone; Teriperatide. Hypocalcaemia associated with Teriperatide is an uncommon side effect. In less than 1 in 1000 teriperatide recipients allergic reactions including dyspnea, urticaria and chest pain have occurred. In patients with risk of osteocarcoma such as Paget's disease, teriperatide use should be avoided. Drug should not be use more than two years ${ }^{[32]}$.

Another major cause of osteoporosis is malnutrition. Calcium intake less than $500 \mathrm{mg} /$ day contributes to 
low bone mass, like wise osteoporosis may also be caused by high intake of caffeine, tea, soft drink, sodium chloride, cigarette smoking, protein and alcohol ${ }^{[33]}$.

Large intake of salt is another major risk factor for development of osteoporosis because excretion of sodium and calcium both increased in urine due to excessive salt intake. Salt intake at a level of $9 \mathrm{~g} /$ day contributes osteoporosis ${ }^{[34]}$.

Like all developed and developing countries, osteoporosis is also very common in Pakistan especially in elderly population. Different treatment options are offered to the patients by their treating physician.

\section{MATERIAL AND METHODS}

Now a day's anti-resorptive and anabolic agents are mainly used to treat osteoporosis. We performed a clinical research investigation and determine the effectiveness of recombinant PTH i.e. parathyroid hormone versus bisphosphonates for the treatment of osteoporosis. The study is aimed to assess the efficacy of recombinant parathyroid hormone versus bisphosphonates for treating osteoporosis on Visual Analogue Scale (VAS).

\section{Study area}

We conducted our research study in Benazir Bhutto Hospital (BBH) Rawalpindi Pakistan, KKT spine center Rawalpindi Pakistan and Amna Hospital Rawalakot Azad Kashmir Pakistan. Data was collected during January 2018 to March 2018 from the patients coming at BBH and KKT spine center.

\section{Data collection}

The total duration of study was 1 year and during the study period a total 60 samples were analyzed randomly. Data was collected using a well-designed questionnaire which are peer reviewed by rheumatologists at the study area i.e. hospital settings. The portion of the collected data reflected some important patient's information such as; name, age, gender, marital status, family history, body mass index, height and weight, OPD number, patient's contact number, address, personal history (it provide us information about personal routines like smoking and alcohol),previous history of trauma (it provide us information about any serious fracture that the patient has experienced in the past), treatment history (it will provide us information about treatment or medicines prescribed to patient in past), co morbidities (it will provide information about existence of two or more disease) and dietary habits recorded on a specially designed Performa or questionnaire.

\section{Hypothesis}

There were two possible hypothesis assumed in this study;

The null hypothesis

The alternative hypothesis

In null hypothesis there was no difference between recombinant parathyroid hormone and bisphosphonates in improving pain on visual analogue scale in osteoporotic patients.

In alternative hypothesis there was difference between recombinant parathyroid hormones and bisphosphonates in improving pain on visual analogue scale in osteoporotic patient.

\section{Study Design}

\section{The inclusion criteria were:}

Patients of age 50 years and above, indoor and outdoor patients and male and female patients present in OPD of Benazir Bhutto hospital or Amna hospital. Filling these criteria was enrolled for study after taking the informed consent from all patients.

\section{Exclusion criteria for this study were:}


Non-randomized controlled trails, pregnant female, poly-trauma patients, patients of age less than 50 years, patient who have undergo recent orthopedic surgery and patient with neurological illness . Patient with these problems were not enrolled for study.

\section{Study parameters}

It include analysis of drugs frequently prescribed (either bisphosphonates or parathyroid hormone), comparison of drugs therapeutic effectiveness, cost and duration of therapy.

Patients follow up was maintained after 3 months to check for therapeutic outcomes of the therapy either with bisphosphonates or rhPTH.

Participants provided their written informed consent to participate in this study. The hospital ethics committee approved this consent procedure.

A number of patients from randomized controlled trail present to OPD with chief complaints of pain in wrist, hip joint, and backache. These complaints were clue of osteoporosis and patients were analyzed and osteoporosis was diagnosed by the help of number of tests such as; DEXA scan (Dual Energy X- Ray Absorptiometry), Vitamin-D test and calcium level test as these test are mostly performed for diagnosis of osteoporosis, and if $T$ - score is $<-2.5$ either in femoral neck, or lumbar spine osteoporosis is confirmed.

\section{Statistical analysis}

Data was analyzed by using different software, data is entered in SPSS version 23.0 (Statistical Package for the Social Sciences), and $\mathrm{P}$ value was significant if $<0.05$. The level of significance is $5 \%$ and power of test is $80 \%$.

\section{RESULT}

Among the approved drug therapies for osteoporosis two classes of drugs are mostly used that are anti resorptive agents (e.g., bisphosphonates, estrogen, calcitonin and selective estrogen receptor modulators) and bone anabolic agent (recombinant human Para-thyroid hormone). Maximum patients in Pakistan were treated with bisphosphonate therapy due to economic status however few patients were prescribed the recombinant parathyroid hormone. There were no significant differences between baseline characteristics of the two drug classes.

A total of 200 patients were examined out of which 120 patients met inclusion criteria. These 120 patients involved 90 female and 30 male patients. Patients were diagnosed with osteoporosis of knee, back and lumber spine. Among 90 female patients11 of them withdrew therapy due to personal reasons as well as due to occurrence of adverse effects and two old ladies died not because of osteoporosis but because of other complications. Among female patients $75 \%$ females were post-menopausal, $27 \%$ were osteopenic and $63 \%$ were osteoporotic. Out of 30 male patients 4 patients gave up due to the adverse effects they experienced. Hence out of 26 male patients who continued therapy $33 \%$ male patients were osteopenic and $77 \%$ were osteoporotic with age above 50 years. $69 \%$ of the osteoporotic patients were treated with bisphosphonates and rest of $31 \%$ was recommended with rhPTH. The clinical design for current study including parameters is given in Table 1.

Table: 1- Study design of patients and parameters

\begin{tabular}{lll}
\hline Parameters of Study & Parameters of Study & Parameters of Study \\
\hline No Of Pt. Examined & 200 & 200 \\
Pt. Met Inclusion Criteria & 120 & 120 \\
Pt. Diagnosis & osteoporosis of knee, back and & osteoporosis of knee, back and \\
Gender & lumber spine & lumber spine
\end{tabular}




\begin{tabular}{lll}
\hline Parameters of Study & Parameters of Study & Parameters of Study \\
\hline No. Of Pt. & 90 & 30 \\
Pt. Withdraw Therapy & 11 & 4 \\
Cause Of Withdrawal & Lack of compliance, side effects & Adverse effects \\
Age Incidence & Post-menopausal 75\% & $>50$ \\
& Pre-menopausal 25 & $77 \%$ \\
Osteoporotic & $63 \%$ & $33 \%$ \\
Osteopenic & $27 \%$ & \\
\hline
\end{tabular}

Patients were kept on follow-up for three months. After three months calcium, vitamin D and DEXA scan tests were again performed. Test reports showed significant increase in Bone Mineral Density of the patients. More significant increase in Bone Mineral Density was observed in patients using Para-thyroid hormone. Lumber spine BMD was greatly increased in patients using Para-thyroid hormone as compared with the patients using bisphosphonate. In the first year of treatment rhPTH increase BMD by $8-9 \%$ while bisphosphonates and estrogen therapy increase BMD 3-5\% during the same time period (Table-2).

Table: 2- Outcome of drug therapy

\begin{tabular}{lllll}
\hline Drugs name & \% of pt. & Lumber spineBMD & Duration of therapy & BMD increased \\
BP & $31 \%$ & - & $1 \mathrm{yr}$. & $3-5 \%$ \\
rhPTH & $69 \%$ & - & $1 \mathrm{yr}$ & $8-9 \%$ \\
\hline
\end{tabular}

However it is of worth, mentioning that recombinant Para-thyroid hormone besides its more effectiveness is prescribed very less in Pakistan because of its increased cost of therapy and is not easily available around the country, whereas bisphosphonates with comparative less effectiveness is prescribed more rapidly due to affordable cost of the entire therapy and easy availability. Due to the developing status of Pakistan, it's unable to formulate recombinant human Para-thyroid hormone subcutaneous injection and hence import the drug.

Table: 3 Showing drugs, dosage form, dose/ strength, frequency, duration, price and availability of two major pharmacological therapies in treatment of osteoporosis.

\begin{tabular}{|c|c|c|c|c|c|c|}
\hline $\begin{array}{l}\text { Major Drugs } \\
\text { Classes }\end{array}$ & $\begin{array}{l}\text { Drugs } \\
\text { included }\end{array}$ & Dosage Form & $\begin{array}{l}\text { Dose/ } \\
\text { Strength }\end{array}$ & $\begin{array}{l}\text { Frequency } \\
\text { and } \\
\text { Duration }\end{array}$ & Price (Rs) & Availability \\
\hline \multirow[t]{5}{*}{ Bisphosphona } & estidronate & Tablet & $\begin{array}{l}20 \mathrm{mg} / \mathrm{kg} \text { for } 2 \\
\text { weeks } \\
10 \mathrm{mg} / \mathrm{kg} \text { for } \\
10 \text { weeks }\end{array}$ & $\begin{array}{l}\text { Once daily } \\
\text { with a total of } \\
12 \text { weeks }\end{array}$ & 290 & Easy \\
\hline & Alendronate & Tablet & 10mg 70mg & $\begin{array}{l}\text { Once Daily } \\
\text { Once weekly } \\
\text { Up to } 10 \text { years }\end{array}$ & 1500 & Easy \\
\hline & Tiludronate & Tablet & $400 \mathrm{mg}$ & $\begin{array}{l}\text { Once daily For } \\
3 \text { months }\end{array}$ & & Easy \\
\hline & Risedronate & Tablet & $\begin{array}{l}5 \mathrm{mg}, 30 \mathrm{mg} \\
35 \mathrm{mg}, 150 \mathrm{mg}\end{array}$ & $\begin{array}{l}\text { Once daily, } \\
\text { Once weekly, } \\
\text { Once monthly }\end{array}$ & $400-700$ & Easy \\
\hline & Ibendronate & $\begin{array}{l}\text { Tablet \& } \\
\text { injectable }\end{array}$ & 100-150mg & $\begin{array}{l}\text { Every } 3 \\
\text { months }\end{array}$ & About 400 & Easy \\
\hline
\end{tabular}


RhPTH Forteo

Subcutaneous $\quad 20 \mathrm{mcg}$

injection

Daily, not

27000

Not easy

more than 2

years

\section{DISCUSSION}

Osteoporosis is considered to be the one of the leading health care problem and hence with our ageing population the prevalence and cost of osteoporosis is increasing ${ }^{[43]}$. Osteoporosis is a common skeletal disease characterized by bone mass reduction and bone micro architecture deterioration including bone fragility and high risk of fractures. Osteoporosis is diagnosed using several tests such as calcium test, vitamin D test and DEXA scan. Among these tests calcium and vitamin D tests are easily affordable however Dual Energy X-rays Absorptiometry (DEXA) scan is not affordable for majority of patients particularly in Pakistan. Furthermore DEXA scan and vitamin D tests are performed following the treatment duration of three months to check whether the treatment strategy is efficacious or not. Among the treatment strategies non-pharmacological treatment as well as pharmacological treatment is available for osteoporosis.

Non-pharmacological treatment includes the life style modifications, tobacco use and excessive use of alcohol and caffeine should be discouraged, promotes intake of balance diet with calcium and vitamin $\mathrm{D}$, regular physical exercise and preventing minor accidents like fall, etc.

Pharmacological treatment of osteoporosis include anti-resorptive agents such as bisphosphonates (Alendronate, Alendronate plus D, Risedronate and Zoledronic acid ), estrogen, calcium and vitamin D supplements, calcitonin and selective estrogen receptor modulators (Raloxifene) and bone anabolic agents including recombinant human Para-thyroid Hormone (rhPTH).

In current study among 120 patients $69 \%$ patients were treated with bisphosphonates and $31 \%$ were treated with rhPTH. However more significant increase in Bone Mineral Density was observed in patients using Para-thyroid hormone. Lumber spine BMD was greatly increased in patients using Para-thyroid hormone as compared with the patients using bisphosphonate. In the first year of treatment rhPTH increase BMD by 8-9\%, while bisphosphonates and estrogen therapy increase BMD 3-5\% during the same time period. The results are comparable with the study conducted by Shen.L (2011) who reported that Parathyroid hormone therapy show areal BMD than therapy with bisphosphonnates. His result showed that as compared to treatment with bisphosphonates lumbar spine BMD increase significantly by use of PTH. Duration and dose dependent increase in hip BMD as compared to bisphosphonates treatment is induced by PTH. Distal radius BMD was significantly lower by treatment with PTH as compared to alendronate.

Bisphosphonates (the anti-resoptive agents) include the drugs etidronate, tiludronate, alendronate, and risedronate, etc. Among them the first generation bisphosphonate i.e.; etidronate $(20 \mathrm{mg} / \mathrm{kg}$ daily for 2 weeks followed by $10 \mathrm{mg} / \mathrm{kg}$ for 10 weeks, a total of 12 weeks followed by 11 to 13 weeks of calcium supplementation) somehow decrease the rate of vertebral fractures but its long term use lead to prolong bisphosphonates retention and impaired mineralization thus causing adverse effects. Tiludronate (400mg daily oral dose for 3 months), alendronate (10mg once daily or $70 \mathrm{mg}$ once weekly and could be used upto 10 years however can be withdrawn after 5 years in some women), and risedronate ( $5 \mathrm{mg}$ once daily taken orally for up to7 years), Ibendronate (100-150mg every 3 months) that are the second and third generation bisphosphonates are much more potent and have greater therapeutic window then etidronate. Etidronate (Disodium); 400mg in a packing of 10 s costs for about $290 \mathrm{Rs}$, Alendronadte(Na); 70mg with a packing of 10 s is available in the cost for about $1500 \mathrm{Rs}$, Risedronate(Na); 35mg in packing of 4s price ranges from 400-700 Rs. Ibendronate; $150 \mathrm{mg}$ comes in price of about 400 Rs (Table.3).

Recombinant human Para-thyroid Hormone (the anabolic agent) include teriparatide with the brand name; Forteo is given as a subcutaneous injection in thigh or abdominal wall, in a dose of 20-mcg daily in a 2.4-ml prefilled delivery device and should not be used for more than 2 years. Price of one Forteo injection $20 \mathrm{mcg}$ in Pakistan is about $27000 \mathrm{Rs}$, which makes it unaffordable for majority of the osteoporotic patients. 
In the current studies it has been observed that risks of vertebral fractures are reduced by long term use of bisphosphonates as compared to those patients who withdrew bisphosphonate therapy earlier. In a previous study conducted by Robert A. Adler (2016) showed that post-menopausal women receiving alendronate for 10 years had fewer clinical vertebral fractures than those who after 5 years were switched to placebo. Whereas few morphometric vertebral fractures were found in women who received zoledronic acid's 6 annual infusions as compared to those who after 3 years were shifted to placebo.

In our study we found rhPTH to be more effective in improving bone architecture and reducing the risk of osteoporosis associated fractures when analyzed in comparison to bisphosphonates. Whereas in a previous study conducted by Hodsman.A (2005) reported fracture risks are significantly reduced by bone mineral architecture improvement as well as overall bone mass enhancement by use of teriperatide. In patients with severe osteoporosis for prevention of fractures PTH should be considered as an alternative therapy to existing anti-resorptive agents. In improving BMD of lumbar spine teriperatide is superior to anti-resorptive therapy (alendronate).

As we have compared bisphosphonates and rhPTH in curing osteoporosis or osteoporotic fractures we find rhPTH to be more efficacious then bisphosphonates but unfortunately due to high cost of rhPTH therapy in Pakistan patients does not afford rhPTH therapy and physician prefer prescribing bisphosphonates keeping in view patient's affordability, as a result of which bisphosphonates are more recommended and prescribed in Pakistan.

\section{CONCLUSION}

Osteoporosis is one of the major health problems worldwide causing bone mass reductions resulting in increased fracture risk. The major treatment strategies include anabolic and anti resorptive drug classes. Recombinant human Parathyroid Hormone is an anabolic agent more effective in the treatment of osteoporotic fractures and increasing Bone Mineral Density (BMD) as compared to Bisphosphonates that are anti resorptive agents. However in Pakistan Bisphosphonate therapy is prescribed more often due to cost effectives and easy availability as compared to rhPTH therapy that is highly expensive and hardly available.

\section{REFERENCES}

1. Shen.L, Xie.X, Su.Y, Luo.C, Zhang.C and Zeng.B. Parathyroid Hormone versus Bisphosphonates Treatment on Bone Mineral Density in Osteoporosis Therapy: A Meta-Analysis of Randomized controlled Trials. PLoS One A Peer-Reviewed Open Access Journal . 2011; 6 (10)

2. Yang.Y, Zhang.X, Zhu.X, Zhang.L, Bao.M, Xian.Y, Wu.J, Mei.L, Li.P. Comparison between Recombinant Parathyroid Hormone (1-34) and elcatonin in treatment of primary osteoporosis. Asian Pacific Journal of Tropical Medicine . 2015;79-84

3. Lane.N. Epidemiology, etiology and diagnosis of osteoporosis. American Journal of Obstetrics and Gynecology. 2006; 194: s3-s11.

4. Tian, MD.L, Xang, Msc.R, Wei, MD.L, Liu, MD.J, Yang, MD.Y, Shao, Msc.F, Ma, Msc.W, Li, Msc.T, Wang, Msc.Y, Guo, Md.T. Prevalence of osteoporosis and related life style and Metabolic factors of postmenopausal women and elderly men: A cross sectional study in Gansu Province, North western of China. Medicine; October 2017, vol.96 (43); pe 8294.

5. Haris.S, Jahan.F, Afreen.A, Ahmed.H and Ahmed.Z. Osteoporosis among Adult Pakistani Population Residing in Karachi Using Quantitative ultrasound Technique. Community Medicine \& Health Education. 2014; 4:4

6. Elliot.M. Prevention and Management of Osteoporosis. The Journal of Pharmacy Society of Wisconsin . 2010; 29-41.

7. Liu.Y, Dvornyk.V, Lu.Y, Shen.H, Lappet.J, Recker.R and Deng.H. A Novel Pathophysiological Mechanism for Osteoporosis suggested by an In Vivo Gene Expression Study of Circulating Monocytes. The Journal of Biological Chemistry. Vol.280 no.32 p.29011-29016.

8. Lin.X, Xiong.D, Peng.Y, Sheng.Z, Wu.X, Wu.X, Wu.F, Yuan.L and Liao.E. Epidemiology and Management of Osteoporosis in People's Republic of China: Current perspectives. Clinical Interventions 
in Aging . 2015; 10: 1017-1033.

9. Dobbs.M, Buckwalter.J, Saltzman.C. "Osteoporosis the Increasing Role of Orthopaedist." The IOWA ORTHOPAEDIC JOURNAL. 1999; 19: 43-52.

10. Faienza.M, Ventura.A, Marzano.F, Cavallo.L. Postmenopausal Osteoporosis: The Role of Immune System Cells. Hindawi Journal of Immunology Research . Vol.2013 (2013) p6.

11. Sozen.T, Ozisik.L, basaran.N. An overview and management of osteoporosis. European Journal of Rheumatology . Vol.4 (1); 2017 Mar.

12. Mirza.F and Canalis.E. SECONDARY OSTEOPOROSIS: PATHOPHYSIOLOGY AND MANAGEMENT. European Journal of Endocrinology . 2015 Sep; 17 (3): R131-R151.

13. Miazgowski.T, Kleevekoper.M, Felsenberg.D, Stepan.J and Szulc.P. Secondary Osteoporosis: Endocrine and Metabolic Causes of Bone Mass Deterioration. Journal of Osteoporosis . Vol. 2012 (2012); 2 pages.

14. Sie-Marie.L. OSTEOPOROSIS: PATHOPHYSIOLOGY AND BONE REMODELING.JOURNAL Society of Obstetricians and Gynecologists Canada. December 1995; 17: 1205-09.

15. Souza.M. OSTEOPOROSIS DIAGNOSIS AND TREATMENT. Revista Brasileria de Ortopedia . 2011 May-Jun; 43(3): 2220-2229.

16. Kostecra.M. The role of healthy diet in the prevention of osteoporosis in postmenopausal period. Pakistan Journal of Medical Sciences . 2014 Jul-Aug; 30(4): 763-768.

17. Cashman.K. Diet, nutrition and Bone health. The Journal of Nutrition. Vol.137(11): 2507S-2512S

18. Sweet.M, Sweet.J, Jeremiah.M, Galazka.S. Diagnosis and Treatment of Osteoporosis. American Family Physician . Vol.79 (3); 2009: 193-200.

19. Reid.I, Grainger.R. Prevention of Osteoporosis. Biomedical and Pharmacology Journal. Issue 17: 6-13.

20. Schrager.S. Osteoporosis Prevention in Primary Care. WISCONSIN MEDICAL JOURNAL. 2003, Vol.102 (3): 52-55.

21. Gabriele.P. Risk factor identification and prevention o osteoporosis in primary care setting. Malta Medical Journal . 18(01): 40-45.

22. Lin.J, Lane.J. Non pharmacologic Management of Osteoporosis to Minimize Fracture Risk. Nature Clinical Practice Rheumatology. 2008; 4(1): 20-25.

23. Cosma.F, Beur.S, LeBoff.M, Lewiecki.E, Tanner.B, Randall.S and Lindsay.R. Clinician's Guide to Prevention and Treatment of Osteoporosis. Osteoporosis International . 2014; 25(10): 2359-3381.

24. Gehrig.L, Lane.J and O'connor.M. Osteoporosis: Management and Treatment strategies for Orthopedic Surgeons. The Journal of Bone and Joint Surgery . 2008; 90: 1362-1374.

25. Fleisch.H. Bisphosphonates in Osteoporosis. European Spine Journal . 2003 Oct; 12 (suppl.2): s142s146.

26. Sinigaglia.L, Varenna.M and Casari.S. Pharmacokinetic Profile of Bisphosphonates in the Treatment of Metabolic bone disorders. Clinical Cases in Mineral and Bone Metabolism . 2007 Jan-Apr; 4(1): 30-36.

27. Russel.R, Rogers.M, Frith.J, Luckman.S, Coxon.F, Benford.H, Croucher.P, Shipman.C and Flerisch.H. The Pharmacology of Bisphosphonates and New Insights into Their Mechanism of Action. Journal of Bone and Mineral Research . 1999; Vol. 14(suppl.2): 53-65.

28. Byun.J, Jang.S, Lee.S, Park.S, Yoon.H, Yoon.B and Ha.Y. The Efficacy of Bisphosphonates for Prevention of Osteoporotic Fractures. An Update Meta-Analysis. Journal of Bone Metabolism . 2011 Feb; 24(1): 37-49.

29. Kennel.K and Drake.M. Adverse Effects of Bisphosphonates: Implication for Osteoporotic Management. Mayo Clinic proceedings . 2009 Jul; 84(7): 632-638.

30. Hodsman.A, Bauer.D, Dempster,D, Dian.L, Hanley.D, Harris.S, Kendler.D, McClung.M, Miller.P, Olszynski.W, Orwoll.E and Yuen.C. Parathyroid Hormone and Teriperatide for the Treatment of Osteoporosis: A Review of the Evidence and Suggested Guidelines for Its Use. Endocrine Reviews . Aug 2005; 26(5): 688-703.

31. Body.J, Gaich.G, Scheele.W, Kulkarni.P, Miller.P, Peretz.A, Dore.R, Rotter.R, Papioannou.A, Cumming.D and Hodsman.A. A Randomized Double-Blind Trial to Compare the Efficacy of Teriperatide 
[Recombinant Human Parathyroid Hormone (1-34)] with Alendronate in Postmenopausal Women with Osteoporosis. The Journal of Clinical Endocrinology and Metabolism. 87(10): 4528-4535.

32. Stroup.J, P-kane.M and Abu-Baker.A. Teriperatide in the Treatment of Osteoporosis. American Journal of Health System. Mar 15, 2005; Vol.65 (6): 532-539.

33. VW B, J Br, The role of nutrition in osteoporosis. Br $j$ biomed science. 1994; 51(3):228-40.

34. AJ. C and FJ. R. Review of risk factors for osteoporosis with particular reference to possible etiological role of dietary salt.DOI . 2000; 38(2-30); 237-253. 